LA-8813-MS

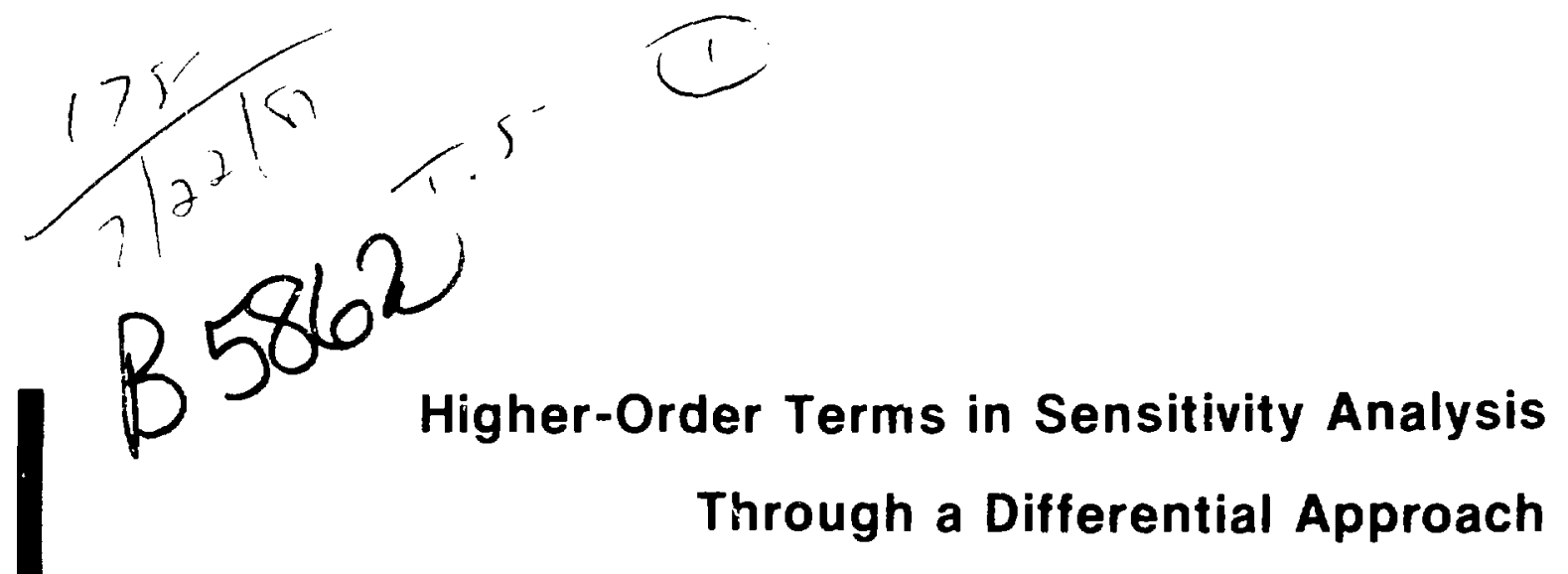

Higher-Order Terms in Sensitivity Analysis

Through a Differential Approach



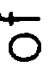

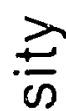

$\stackrel{\frac{1}{5}}{\supset}$ 


\title{
Higher-Order Terms in Sensitivity Analysis Through a Differential Approach
}

\author{
Arie Dubi *
}

Donald J. Dudziak 
CONTENTS

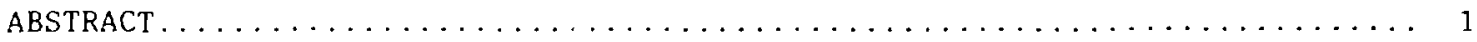

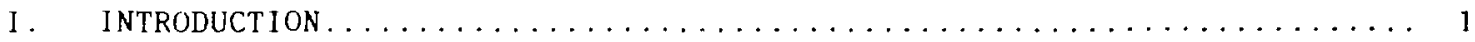

I1. GENERAL DISCUSSION AND DEFINITIONS OF THE THEORY OF FUNCTIONALS...... 2

II I. THE TARGET RESPONSE FUNCTIONAL $\ldots \ldots \ldots \ldots \ldots \ldots \ldots \ldots \ldots \ldots \ldots \ldots \ldots$

IV. HIGHER-ORDER TERMS OF THE TAYLOR SERIES $\ldots \ldots \ldots \ldots \ldots \ldots \ldots \ldots \ldots \ldots \ldots$

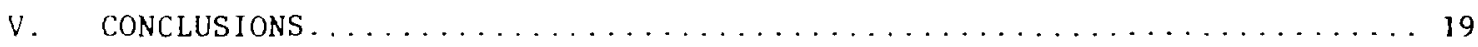

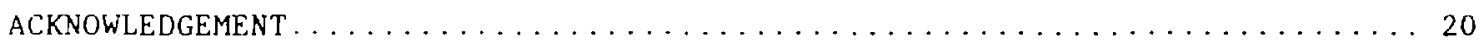

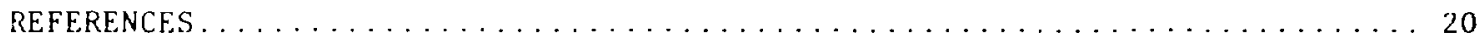


HIGHER-ORDER TERMS IN SENSITIVITY ANALYSIS THROUGH A DIFFERENTIAL APPROACH

by

Arje Dubi and Donald J. Iudziak

Theoretical Division

\begin{abstract}
A difterential approach to sensitivity analysis has Leen developed that eliminates some difficulties existing in previous work. The new development leads to simple explicit expressions for the first-order perturbation as well as any higher-order terms. The higher-order terms are dependent only on differentials of the transport operator, the unperturbed flux, the adjoint flux, and the unperturbed Green's function of the system.
\end{abstract}

1. INTRODUCTION

In a recent publication oblow suggested a differential approach to the problem of sensitivity analysis. The basic features of this approach are as follows. The target response $R$ is presented as an integrai functional of the form

$R=\int F|\phi(\bar{x}), \Sigma(\bar{x})| d \bar{x}$,

where $\varphi(\bar{x})$ is the flux at $\bar{x}$ and $\Sigma(\bar{x})$ is the "data tield." A small increment $\delta \Sigma(\bar{x})$ is added at each point $\bar{x}$ to the data field and the new target response is written as a Taylor expansion of the increment in the form

$R[\Sigma(\bar{x})+\delta \Sigma(\bar{x})]=R[\Sigma(\bar{x})]+\int_{\bar{x}} \frac{d R}{d \Sigma(\bar{x})} \delta \Sigma(\bar{x}) d \bar{x}+\frac{1}{2 !} \int_{\frac{x}{x}} \frac{d^{2} R}{d \Sigma(\bar{x})^{2}}[\delta \Sigma(\bar{x})]^{2} d \bar{x}+\ldots$ 
Taking a first-order approximation yields

$\delta R=\int_{\bar{x}} \frac{d R}{d \Sigma(\bar{x})} \delta \Sigma(\tilde{x}) d \bar{x}$,

and the idea then is to calculate $d R / d \Sigma(\bar{x})$ and to show that Eq. (3) gives the same result as that obtained by first-order perturbation theory.

Three questionable points arise concerning the above representation. First, the meaning of the derivative $\mathrm{dR} / \mathrm{d} \bar{L}(\bar{x})$ is not clear. Since $R$ is a functional of the function $\Sigma(\bar{x})$ and not a function of the variable $\Sigma$, it is not clear how such a derivative can be defined at a point $\bar{x}$ of zero measure. The second questionable point concerns the obvious dimensional inconsistency in Eqs. (2) and (3). One may note that the left-hand side of Eq. (3) has the dimension of $R$, whereas the right-hand side has the dimension of $R$ inultiplied oy the dimension of the phase-space variable. The third point concerns the fact that it is not at all clear that the form of the Taylor expansion of Eq. (2) can be assumed to hold without a proof. It will be later shown that, in fact, in the general case of a functional of the form of Eq. (1), where the functions $\phi(\bar{x})$ and $\Sigma(\bar{x})$ are externally related by a differentia or integredifferential equation, it is impossible to assume a Taylor expansion of the form of Eq. (2), so a dicferent, more general, form should be adopted.

In the following, we suggest a somewhat different development of the differential approach to sensitivity that avoids the above-mentioned difficulties. Further, the suggested development leads to explicit expressions for higher-order terms in the Taylor expansion of the target response $R$. We arrive at a general explicit form of the nth-order term which involves no differentials of the flux or the adjoint flux.

\section{GENERAL DISCUSSION AND DEFINITIONS OF THE THEORY OF FUNCTIONALS}

In its general sense the roncept of a functional is an extension of the concept of a function. If we have two sets of values $x$ and $y$, we say that $y$ is a function of $x$, if to every value of $x$ corresponds a value of $y .^{\dagger}$

†n general, $x$ can be the phase space variable $\bar{x}$ used above, but for simplicity we use the scalar notation. 
Similarly, if we have a set of functions $\{\rho(x)\}$ comprising à subset of a normed vector space, ${ }^{2}$ where each function is defined on the interval ( $\left.a, b\right)$ and we have a set of values $J$, then we say that $J$ is a functional of $\rho(x)$, if to every function $\rho(x)$ corresponds one of the values $j$. ? The value of $J$ will depend, in general, on all the values of the function within its range $(a, b)$. This relation between the functional $J$ and the set of functions $\{\rho(x)\}$ is denoted by

$J=\underset{a}{J[\rho(x)]}$.

The derivative of $J$ with respect to the function $p$ at the point $x$ must, naturally, be related both to an increment of $\rho$ and to a volume element of . in which the increment of $\rho$ is introduced. The derivative is this defind in the following manner. 4 Let $\xi$ be a fixed point in the interval (a,b) and let $h$ be the amplitude of an interval centered at $\xi$; that is. $(\xi-h / 2, \xi+h / 2)$. $^{\dagger}$ t us define an increment $t o p(x)$ of the form $\theta(x)=\varepsilon \cdot \delta \rho(x)$, whece $\varepsilon$ is a posituve real number and $\delta \rho(x)$ is a function in the same normed vector space that is nonzero only inside the interval $(\xi-h / 2, \xi+h / 2)$. Next we define the quantity

$\sigma=\varepsilon \cdot \int_{\xi-h / 2}^{\xi+h / 2} \delta \rho(x) d x$

The first derivative of the functiond J with respect to the function $p(x)$ at the point $\xi$ is now defined as

$$
\begin{aligned}
& \stackrel{a}{\rho}(x)+b \cdot \delta \rho(x)]-J[\rho(x)] \\
& \left.J^{\prime} \mid \rho(x) ; \xi\right]=\operatorname{limit}_{\substack{\varepsilon \rightarrow 0 \\
h \neq 0}} \quad b \quad \sigma^{-\ldots} \ldots
\end{aligned}
$$

${ }^{\dagger} \mathrm{f} \rho(\bar{x})$ is defined on a normed vector space, the interval can be replaced by a ball of radius $h$ centered at $\xi$. 
Note that this definition accounts for the ratio of the change in the functional and a change in the function, where both the change in the function and the interval. in which this change is applied shrink to zero. This definition is considerably different than the definition of derivatives in elementary function theory. The dimensions of this derivative are those of $\mathrm{J}$ divided by the dimension of $\rho$ and $x$ and cannot be written as $d J / d \rho$ without causing a potential misunderstanding, because $\mathrm{dJ} / \mathrm{d} \rho$ may be interpreted as

$\operatorname{limit}[J(\rho+\Delta \rho)-J(\rho)] / \Delta \rho$, $\Delta \rho \rightarrow 0$

which is meaningless in the context of functionals. Next we define the Gâteaux variation ${ }^{5}$ of a functional. Let $\delta \rho(x)$ be an increment to $\rho(x)$ in (a,b) and $\varepsilon$ a positive real number. If there exists a functional $\delta J \mid \rho(x) ; \delta \rho(x)]$ such that

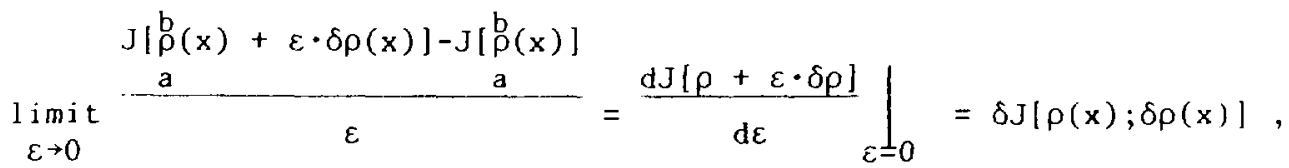

then $\delta J[\rho ; \delta \rho]$ is called the Gâteaux variation of $J$ at the function $\rho$. It the variation functional is continuous with respect to $\rho(x)$ (Ref. 6) and is linear in $\delta \rho(x)$, it is then denoted by $\mathrm{dJ}[\rho ; \delta \rho]$ and called the Frêchet differential of $J$ In the following, the convention is used that when a functional $J$ depends on ail the values of a function $\rho(x)$ in an interval (a,b), it will be denoted by $J[\rho]$ without a mention of the variable $x$.

The concept of a Taylor series expansion of a functional may row be introduced as follows. Consider the functional $J[\rho+\varepsilon \cdot \delta \rho]$. This functional can be viewed, for a fixed function $\rho$ and a fixed increment $\delta \rho$; as a regular elementary function of $\varepsilon$, namely, $f(\varepsilon)$. Thus we may write

$J[\rho+\varepsilon \cdot \delta \rho]=f(\varepsilon)$

and

$J[\rho+\delta \rho]=f(1)$ 
Now $f(1)$ can be expanded in a Taylor series around $\varepsilon=0$ in the usual form

$$
f(1)=f(0)+\left.\frac{d f}{d \varepsilon}\right|_{\varepsilon=0}+\left.\frac{1}{2 !} \frac{d^{2} f}{d \varepsilon^{2}}\right|_{\varepsilon=0}+\ldots
$$

Equation (9) represents the most general form of a Taylor series expansion (T.S.E) of a functional. One can easily identify the first-order term in the T.S.E as the Gâteaux variation of the functional.

Consider now a functional of the form

$$
J[\rho]=\int_{a}^{b} F[\rho(x)] d x,
$$

where $F$ is some function of $\rho$. For such a functional it is possible to relate the derivative in Eq. (6) to the Gâteaux variation in Eq. (7) and, hence, to the T.S.E. Consider the expression

$$
\begin{aligned}
\left.\int_{a}^{b} J \cdot \rho ; \xi\right] \cdot \delta \rho(\xi) d \xi & =\int_{a}^{b} \operatorname{limit}_{\substack{\varepsilon \rightarrow 0 \\
h \rightarrow 0}}^{b} \frac{J[\rho+\varepsilon \cdot \delta \rho]-J[\rho]}{G} \cdot \delta \rho(\xi) d \xi \\
& =\int_{a}^{b} \operatorname{limit}_{\substack{\varepsilon \rightarrow 0 \\
h \rightarrow 0}} \frac{\{\{F[\rho(x)+\varepsilon \cdot \delta \rho(x)]-F[\rho(x)]\} d x}{\sigma} \cdot \delta \rho(\xi) d \xi
\end{aligned}
$$

Because, by the definition of the derivative, $\delta \rho(x)$ is nonzero only in the interval $(\xi-h / 2, \xi+h / 2)$, the integrand of the integral over $x$ will also be nonzero only in that interval. Thus we may write

$$
\begin{aligned}
& \xi+h / 2 \\
& \int_{a}^{b} J^{\prime}[\rho ; \xi] \cdot \delta \rho(\xi) d \xi=\int_{a}^{b} \operatorname{limit}_{\substack{\varepsilon \rightarrow 0 \\
h \rightarrow 0}} \frac{\int_{-h / 2}\{F[\rho(x)+\varepsilon \cdot \delta \rho(x)]-F[\rho(x)]\} d x}{\varepsilon \cdot \int_{\xi-h / 2}^{\xi+h / 2} \delta \rho(x) d x} \delta \rho(\xi) d \xi .
\end{aligned}
$$


Taking now the limit as $h \rightarrow 0$ and exchanging the limiting of $\varepsilon \rightarrow 0$ with the integration over $\xi$, we obtain

$$
\begin{aligned}
& \int_{\mathbf{a}}^{\mathrm{b}} J^{\prime}[\rho ; \xi] \cdot \delta \rho(\xi) \mathrm{d} \xi=\operatorname{limit}_{\varepsilon \rightarrow 0} \int_{\mathbf{a}}^{\mathrm{b}} \frac{\{F[\rho(\xi)+\varepsilon \cdot \varepsilon \rho(\xi)]-F[\rho(\xi)]\}}{\varepsilon} \mathrm{d} \xi \\
& =\left.\frac{d J[\rho+\varepsilon \cdot \delta \rho]}{d \varepsilon}\right|_{\varepsilon=0}=\delta J[\rho: \delta \rho] \text { (the Gâteaux variation). }
\end{aligned}
$$

Using Eq. (12) for the first-order term of Eq. (9) and following the same. procedure for higher-order terms, it can be shown ${ }^{7}$ that the T.S.E for the functional of the type of Eq. (10) can be written in terms of the functional derivatives in the form

$$
\begin{aligned}
J[\rho+\delta \rho]= & J[\rho]+\int_{a}^{b} J^{\prime}\left[\rho ; \xi_{1}\right] \cdot \delta \rho\left(\xi_{1}\right) d \xi_{1} \\
& +\frac{1}{2 !} \int_{a}^{b} \int_{a}^{b} J^{\cdots}\left[\rho ; \xi_{1}, \xi_{2}\right] \cdot \delta \rho\left(\xi_{1}\right) \cdot \delta \rho\left(\xi_{2}\right) d \xi_{1} d \xi_{2} \\
& +\frac{1}{3 !} \int_{a} \int_{a}^{b} \int_{a}^{b} J\left[\rho ; \xi_{1}, \xi_{2}, \xi_{3}\right] \cdot \delta \rho\left(\xi_{1}\right) \cdot \delta \rho\left(\xi_{2}\right) \cdot \delta \rho\left(\xi_{3}\right) d \xi_{1} d \xi_{2} d \xi_{2}+\ldots
\end{aligned}
$$

This form somewhat resembles Eq. (2). However, it is essential at this point to note that Eq. (12) was derived only for functionals of the form of Eq. (10). The passage from the basic form of Eq. (9) to Eq. (13) is made possible by virtue of the fact that, in the proof of Eq. (11), it was possible. to shrink the limits of integration in the numerator from the interval $(a, b)$ to the interval $(\xi-h / 2, \xi+h / 2)$, and thereby explicitly take the iimjt when $h \rightarrow 0$. This procedure is applicable because, for the specific form of a functional of Eq. (10), a change in the function $\rho(x)$ in the interval $(\xi-h / 2, \xi+h / 2)$ brings about a change in the contribution to the functional only in that interval and nowhere else. These conditions will not hold for the type of functionals to which the target response $R$ belongs, as is shown below. 
I I I. THE TARGET RESPONSE FUNCTIONAL

The target response functional can be written in the form

$R=\int_{D} F[\bar{x}, \phi(\bar{x}), \Sigma(\bar{x})] d \bar{x}=R[\phi, \Sigma]$,

where $\phi(\bar{x})$ and $\Sigma(\bar{x})$ are externally related, for example, by an integrodifferential equation of the form of the transport equation

$\mathrm{L}[\overline{\mathrm{x}}, \Sigma(\overline{\mathrm{x}})] \cdot \phi(\overline{\mathrm{x}})=\mathrm{S}(\overline{\mathrm{x}})$

In this type of functional, $\Sigma(\bar{x})$ is a known function (sometimes referred to is a control function ${ }^{8}$ ) and $\phi(\bar{x})$ is an unknown function. Two important facts should be noted. The integration in Eq. (14) may be taken over a limited sutset of the phase space (the detector region, $D)$; and, in the general sense, $\phi\left(\bar{x}_{0}\right)$ at every given point $\bar{x}_{0}$ is itself a functional of $\Sigma(\bar{x})$. Thus, an increment $\delta \Sigma(\bar{y})$ in a small region around $\bar{y}$ will affect the contribution to $R$ not only in that region $\{$ as was the case with the functional of Eq. (10)l, but everywhere in $D$. This occurs through its effect on the flux, $\phi(\bar{x})$. In fact, $\bar{y}$ may be outside $D$ and the functional will still be affected. It is because of this effect that the shrinking of the limits of integration, as used to de.ive Eq. (11), cannot be repeated for $R$ and the T.S.E for $R$ cannot be assumed to be in $\mathrm{tl}$ a form of Eq. (13). To get a T.S.E for $\mathrm{R}$ we must use the more basic form of Eq. (9).

A general expression for the first-order term $\left.d \tilde{f} / d \varepsilon{ }_{\varepsilon}\right\rfloor_{0}$ for a functional of the form of Eq. (14) is derived in Ref. (8). Let $\varepsilon \cdot \delta \Sigma(\bar{x})$ be an increment to $\Sigma(\bar{x})$ at any point $\bar{x}$. Because $\phi$ is a functional of $\Sigma(\bar{x})$, we will denote it by $\phi[\bar{x}, \Sigma]$. Then

$\left.\frac{\mathrm{df}}{\mathrm{d} \varepsilon}\right|_{\varepsilon=0}=\left.\frac{\mathrm{d}}{\mathrm{d} \varepsilon} \mathrm{R}[\phi(\overline{\mathrm{x}}, \Sigma+\varepsilon \cdot \delta \Sigma), \Sigma+\varepsilon \cdot \delta \Sigma]\right|_{\varepsilon=0}=\left.\int_{\mathrm{D}} \frac{\mathrm{d}}{\mathrm{d} \varepsilon} \mathrm{F}[\overline{\mathrm{x}}, \phi(\overline{\mathrm{x}}, \Sigma+\varepsilon \cdot \delta \Sigma), \Sigma+\varepsilon \cdot \delta \Sigma]\right|_{\varepsilon=0} \mathrm{~d} \overline{\mathrm{x}}$.

The above equality is true assuming that $F[\bar{x}, \phi, \Sigma]$ is continuous with respect to its three variables and has continuous first-order partial derivatives. The derivative $\frac{d F}{d \varepsilon}$ can now be calculated by the chain rule of differentiai calculus for each fixed value of $\bar{x}$, yielding 
$\left.\frac{\mathrm{d}}{\mathrm{d} \varepsilon} \mathrm{F}[\overline{\mathrm{x}}, \phi(\overline{\mathrm{x}}, \Sigma+\varepsilon \cdot \delta \Sigma), \Sigma(\overline{\mathrm{x}})+\varepsilon \cdot \delta \Sigma(\overline{\mathrm{x}})]\right|_{\varepsilon=0}=\left.\frac{\partial F}{\partial \phi}[\overline{\mathrm{x}}, \phi(\overline{\mathrm{x}}, \Sigma), \Sigma(\overline{\mathrm{x}})] \cdot \frac{\mathrm{d}}{\mathrm{d} \varepsilon} \phi(\overline{\mathrm{x}}, \Sigma+\varepsilon \cdot \delta \Sigma)\right|_{\varepsilon=C}$

$$
+\frac{\partial F}{\partial \Sigma}[\bar{x}, \phi(\bar{x}, \Sigma), \Sigma(\bar{x})] \cdot \delta \Sigma(\bar{x})
$$

where the derivatives $\frac{\partial F}{\partial \phi}$ and $\frac{\partial F}{\partial \Sigma}$ are ordinary derivatives of the function $\mathrm{F}(\overline{\mathrm{x}}, \phi, \check{z})$. Substituting $\mathrm{Eq}$. (17) into (16) we obtain

$$
\begin{aligned}
\left.\frac{\mathrm{df}}{\mathrm{d} \varepsilon}\right|_{\varepsilon=0}= & \left.\int_{D} \frac{\partial F[\bar{x}, \phi(\bar{x}, \Sigma), \Sigma(\bar{x})]}{\partial \phi} \cdot \frac{\mathrm{d}}{\mathrm{d} \varepsilon} \phi(\overline{\mathrm{x}}, \Sigma+\varepsilon \cdot \delta \Sigma)\right|_{\varepsilon=0} \mathrm{~d} \overline{\mathrm{x}} \\
& +\int_{\mathrm{D}} \frac{\partial \mathrm{F}[\overline{\mathrm{x}}, \phi(\overline{\mathrm{x}}, \Sigma), \Sigma(\overline{\mathrm{x}})]}{\partial \Sigma} \delta \Sigma(\overline{\mathrm{x}}) \mathrm{d} \overline{\mathrm{x}} .
\end{aligned}
$$

Taking the first-order approx mation to $f(1)$, we can immediately see that

$\delta R=f(1)-f(0) \cong d f / d \varepsilon_{\varepsilon=0}=\delta R_{1}$.

Thus, $\delta R_{1}$, as given in Eq. (18), represents the first-order approximation to $\delta R$.

Looking at Eq. (18), we may notice that the second term on the right-hana side represents the "direct effect"; that is, the change in the target response as a resu,t of the change in $\Sigma(\bar{x})$ only, without the resulting change in the flux. This term has the form of Eq. (11) because a change in $\Sigma(\bar{x})$ in a given interval contributes to the change in $R$ directly in that interval. Thus, as far as this second term is concerned, we have a situation similar to the one for the functional of Eq. (10). On the other hand, the first term of the right-hand side of Eq. (18) accounts for the indirect effect of the change in the flux all over the detector range $D$ caused by a specified change $\delta \Sigma(\bar{y})$ in any subsei of the phase space. The subset may or may not include the detector range or parts of it. To get an explicit expression for this term we need to calculate

$\frac{\mathrm{d}}{\mathrm{d} \varepsilon} \phi[\overline{\mathrm{x}}, \Sigma+\varepsilon \cdot \delta \Sigma] \underset{\varepsilon=0}{\left.\right|_{0}}=\operatorname{limit}_{\varepsilon \rightarrow 0} \frac{\phi[\overline{\mathrm{x}}, \Sigma+\varepsilon \cdot \delta \Sigma]-\phi[\mathrm{x}, \Sigma]}{\varepsilon}$, 
where Eq. (20) simply follows the definition of the Gâteaux variation of a functional.

To calculate $d \phi / d \varepsilon{ }_{\varepsilon} \perp_{0}$, we take the following steps. $\phi(\bar{x}, \bar{\Sigma}+\varepsilon \cdot \delta \Sigma)$ is the is the solution of the transport equation

$L[\bar{x}, \Sigma(\bar{x})+\varepsilon \cdot \delta \Sigma(\bar{x})] \phi(\bar{x}, \Sigma+\varepsilon \cdot \delta \Sigma)=S(\bar{x})$

where $L$ is the transport operator and $S(\bar{x})$ is the inhomogeneous source term. Let $\phi^{*}\left(\bar{x}, \bar{x}_{0}, \Sigma+\varepsilon \cdot \delta \Sigma\right)$ be the solution of the adjoint operator equation

$L^{*}[\bar{x}, \Sigma(\bar{x})+\varepsilon \cdot \delta \Sigma(\bar{x})] \phi^{\dot{x}}\left(\bar{x}, \bar{x}_{0}, \Sigma+\varepsilon \cdot \delta \Sigma\right)=\delta\left(\bar{x}-\bar{x}_{0}\right)$

where $L^{*}$ is the operator adjoint to $L$ and $\delta\left(\bar{x}-\bar{x}_{0}\right)$ is a phase-space delta function: By virtue of the definition of the adjoint operator and adjoint equation, we have. using Eqs. (21) and (22),

$\int \phi^{*}\left(\bar{x}, \bar{x}_{0}, \Sigma+\varepsilon \cdot \delta \Sigma\right) L[\bar{x}, \Sigma(\bar{x})+\varepsilon \cdot \delta \Sigma(\bar{x})] \phi(\bar{x}, \Sigma+\varepsilon \cdot \delta \Sigma) d \bar{x}$

$\left.=\int \phi \dot{\bar{x}}, \bar{x}_{0}, \Sigma+\varepsilon \cdot \delta \Sigma\right) S(\bar{x}) d \bar{x}=\int \phi(\bar{x}, \Sigma+\varepsilon \cdot \delta \Sigma) L(\bar{x}, \Sigma+\varepsilon \cdot \delta \Sigma) \phi\left(\bar{x}_{,} \bar{x}_{0}, \Sigma+\varepsilon \cdot \delta \Sigma\right) d x$

$=\int \phi(\bar{x} \cdot \tau+\varepsilon \cdot \delta \Sigma) \delta\left(\bar{x}-\bar{x}_{0}\right) d \bar{x}=\phi\left(\bar{x}_{0}, \Sigma+\varepsilon \cdot \delta \Sigma\right)$.

$\phi *\left(\bar{x}^{*}, \bar{x}_{0}, \Sigma+\varepsilon \cdot \delta \Sigma\right)$ may be recognized as the adjoint Green's function giving the flux at $\bar{x}_{0}$ resulting from a unit point source at $\bar{x}$. (This definition is somewhat djfferent than the usual definicion of the adjoint flux as being the detector response resulting from a unit point source at $\bar{x}$, unless one considers a unit response function.) We may then write from Eq. (23)

$\phi\left(\bar{x}_{0}, \Sigma+\varepsilon \cdot \delta \Sigma\right)=\int \phi^{*}\left(\bar{x}, \bar{x}_{0}, \Sigma+\varepsilon \cdot \delta \Sigma\right) S(\bar{x}) d \bar{x}$.

[hus,

$\left.\frac{\mathrm{d}}{\mathrm{d} \varepsilon} \phi\left(\overline{\mathrm{x}}_{0}, \Sigma+\varepsilon \cdot \delta \Sigma\right)\right|_{\varepsilon=0}=\left.\int \frac{\mathrm{d} \phi \overline{\mathrm{x}}^{*}\left(\overline{\mathrm{x}}, \overline{\mathrm{x}}_{0}, \Sigma+\varepsilon \cdot \delta \Sigma\right)}{\mathrm{d} \varepsilon}\right|_{\varepsilon=0} \mathrm{~S}(\overline{\mathrm{x}}) \mathrm{d} \overline{\mathrm{x}}$. 
To proceed we take the derivative with respect to $\varepsilon$ at $\varepsilon=0$ of $E_{i}$. (22), yielding ${ }^{\dagger}$

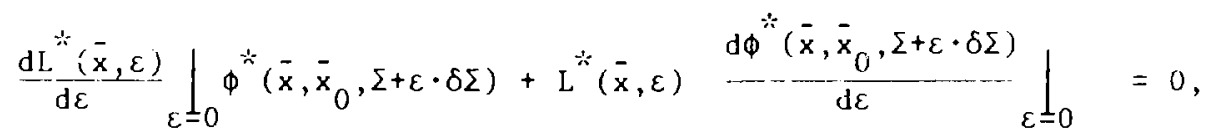

which can be written as

$\left.L^{*}(\bar{x}, \Sigma) \frac{d \phi^{*}\left(\bar{x}, \bar{x}_{0}, \Sigma+\varepsilon \cdot \delta \Sigma\right)}{d \varepsilon}\right|_{\varepsilon=0}=-\left.\frac{d L^{*}}{d \varepsilon} \frac{\left(\bar{x}_{2} \varepsilon\right)}{d \varepsilon}\right|_{\varepsilon=0} \phi^{*}\left(\bar{x}_{0}, \bar{x}_{0}, \bar{z}\right)$

Returning now to Eq. (25) and using Eq. (21), we get

$\left.\frac{\mathrm{d} \phi *\left(\overline{\mathrm{x}}, \overline{\mathrm{x}}_{0}, \Sigma+\varepsilon \cdot \delta \Sigma\right)}{\mathrm{d} \varepsilon}\right|_{\varepsilon=0} S(\overline{\mathrm{x}}) \mathrm{d} \overline{\mathrm{x}}=\left.\frac{\mathrm{d} \phi^{*}\left(\overline{\mathrm{x}}^{\prime}, \overline{\mathrm{x}}_{0}, \bar{\Sigma}+\varepsilon \cdot \delta \Sigma\right)}{\mathrm{d} \varepsilon}\right|_{\varepsilon=0} L(\overline{\mathrm{x}}, \Sigma) \phi(\overline{\mathrm{x}}, \Sigma) \mathrm{d} \overline{\mathrm{x}}$.

Using the definition of the adjoint operator, this equals

$\phi(\bar{x}, \Sigma) L^{*}\left(x^{-}, \Sigma\right) \frac{\mathrm{d} \phi *\left(\bar{x}, \bar{x}_{0}, \Sigma+\varepsilon \cdot \delta \Sigma\right)}{d \varepsilon}-\frac{1}{d}-$

and by Eq. (27) we can then write Eq. (25) as

$\left.\frac{\mathrm{d}}{\mathrm{d} \varepsilon} \phi\left(\overline{\mathrm{x}}_{\mathrm{U}}, \Sigma+\varepsilon \cdot \delta \Sigma\right)\right|_{\varepsilon=0}=-\left.\int \phi(\overline{\mathrm{x}}, \Sigma) \frac{\mathrm{dL}{ }_{\mathrm{N}}^{*}(\overline{\mathrm{x}}, \varepsilon)}{\mathrm{d} \varepsilon}\right|_{\varepsilon=0} \phi *\left(\overline{\mathrm{x}}, \overline{\mathrm{x}}_{0}, \Sigma\right) \mathrm{d} \overline{\mathrm{x}}$.

Substituting the right-hand side of Eq. (28) into the first term on the righthand side of Eq. (18) gives the indirect first-order approximation

$\delta R_{1, \text { indirect }}=-\left.\int_{D} \int_{\bar{x}} \frac{\partial F\left[\bar{x}_{0}, \phi\left(\bar{x}_{0}, \Sigma\right), \Sigma\left(\bar{x}_{0}\right)\right]}{\partial \phi} \phi(\bar{x}, \Sigma) \frac{d L^{*}(\bar{x}, \varepsilon)}{d \varepsilon}\right|_{\varepsilon=0} \phi^{*}\left(\bar{x}, \bar{x}_{0}, \Sigma\right) d \bar{x}^{\prime} d \bar{x}_{0}$.

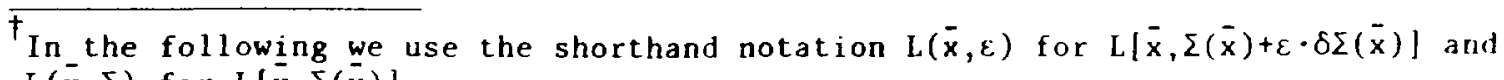
$L(\bar{x}, \Sigma)$ for $L[\bar{x}, \Sigma(\bar{x})]$. 
We may now carry out the integration over $\bar{x}_{0}$. In the usual case where

$F\left(\bar{x}_{0}, \phi, \Sigma\right)=\phi\left(\bar{x}_{0}\right) \cdot \Sigma_{r}\left(\bar{x}_{0}\right), \dagger$

then clearly

$\frac{\partial F}{\partial D}=i_{r}\left(\bar{x}_{0}\right)$

Using Eq. (31) we get

$\int_{x_{0} \varepsilon D} \Sigma_{r}\left(\bar{x}_{0}\right) \phi \dot{x}\left(\bar{x}_{0}, \Sigma\right) d \bar{x}_{0}=\phi^{*}(\bar{x}, \Sigma)$

where now $\phi^{*}(\bar{x}, \Sigma)$ is the usual adjoint flux that is the solution of the equation $L^{*} \phi^{*}=\Sigma_{v}$. With the use of Eq. (32), Eq. (29) can be written as

$\delta \mathrm{R}_{1, \text { indirect }}=-\left.\int \phi(\overline{\mathrm{x}}, \Sigma) \quad \frac{\mathrm{dL} \dot{*}\left(\bar{x}_{2} \varepsilon\right)}{\mathrm{d} \varepsilon}\right|_{\varepsilon=0} \phi^{*}(\overline{\mathrm{x}}, \Sigma) \mathrm{d} \overline{\mathrm{x}} ;$

and because, for the transport operator,

$\left[\frac{d L *(\bar{x}, \varepsilon)}{d \varepsilon}\right]=\frac{d L}{d *}(\bar{x}, \varepsilon)$

we get

$\delta R_{1, \text { indirect }}=\left.\int_{\bar{x}} \phi^{*}(\bar{x}, \Sigma) \frac{\mathrm{dL}(\overline{\mathrm{x}}, \varepsilon)}{\mathrm{d} \varepsilon}\right|_{\varepsilon=0} \phi(\bar{x}, \Sigma) \mathrm{d} \overline{\mathrm{x}}$,

which is identical with the result of first-order perturbation theory. ${ }^{9}$ Note that Eq. (29) has a very basic meaning. The integration over $\bar{x} y i e l d s$

$\bar{T}_{\mathrm{r}}\left(\overline{\mathrm{x}}_{0}\right)$ may be some partial cross section accounting for a specific interaction. 
the variation of the flux at the point $\bar{x}_{0}$ resulting from a change in the data field $\Sigma(\bar{x})$ all over the phase space. This transforms the change in the data field into a local change in the flux in the detector range. This is the most essential part of the variation of the target response. The integration over $\bar{x}_{0}$ merely folds the variation in the flux with any specific detector response function.

\section{HIGHER-ORDER TERMS OF THE TAYLOR SERIES}

At this point one would like to pose the question of whether it is pussibi.. to calculate higher-order terms in the series and whether such higher-order tern. can be expressed in terms of the unperturbed flux and differentials of the operator only. The rest of the discussion will show that this is inderi possible provided one knows the detailed Green's function of the given systent Because the perturbation in the flux is the most difficult and essential part w the change in the target response, we first consider the perturbed flux ai: point $\bar{x}_{0}$, namely, $\phi\left(\bar{x}_{0}, \Sigma+\delta \Sigma\right)$. We will seek to develop a Taylor series for thw perturbed flux, of which the first-order term, when substituted in the betector response, yields the result obtained in the former section (first-order perturbation theory) and the higher terms are tigher-order terms of the perturbation.

Noting that the $\mathrm{flux}$ is also a functional of $\Sigma(\bar{y})$, we may refer $\ldots$ $\phi\left(\bar{x}_{0}, \Sigma+\varepsilon \cdot \delta \Sigma\right)=g(\varepsilon)$ as a function of $\varepsilon$ (for every fixed $\bar{x}_{0}$, $\Sigma$ and $\delta \Sigma$ ) and then [see Eq. (9)] look at $g(1)$ as a Taylor series expansion around $\varepsilon=$

$\phi\left(\bar{x}_{0}, \Sigma+\delta \Sigma\right)=g(1)=g(0)+\left.\frac{d g}{d \varepsilon}\right|_{\varepsilon=0}+\left.\frac{1}{2 !} \frac{d^{2} g}{d \varepsilon^{2}}\right|_{\varepsilon=0}+\ldots$

The first two terms on the right-hand side are already known, the first being the unperturbed flux at $\bar{x}_{0}$. The second term can be obtained using Eg. (28) and the adjoint properties; namely,

$$
\left.\frac{\mathrm{d} \phi\left(\bar{x}_{0}, \Sigma+\varepsilon \cdot \delta \Sigma\right)}{\mathrm{d} \varepsilon}\right|_{\varepsilon=0}=-\left.\int \phi\left(\bar{x}^{*}, \bar{x}_{0}, \Sigma\right) \quad \frac{\mathrm{dL}(\overline{\mathrm{x}}, \Sigma+\varepsilon \cdot \delta \Sigma)}{\mathrm{d} \varepsilon}\right|_{\varepsilon=0} \phi(\overline{\mathrm{x}}, \Sigma) \mathrm{dx}=\left.\frac{\mathrm{d} g}{\mathrm{~d} \varepsilon}\right|_{\varepsilon=0} .
$$


By using Eq. (24), the next term in the series will be

$$
\left.\frac{d^{2} g}{d \varepsilon^{2}}\right|_{\varepsilon=0}=\left.\frac{d^{2} \phi\left(\bar{x}_{0}, \Sigma+\varepsilon \cdot \delta \Sigma\right)}{d \varepsilon^{2}}\right|_{\varepsilon=0}=\left.\frac{d^{2} \phi^{*}\left(\bar{x}_{0}, \bar{x}_{0}, \Sigma+\varepsilon \cdot \delta \Sigma\right)}{d \varepsilon^{2}}\right|_{\varepsilon=0} S(\bar{x}) d \bar{x} .
$$

To ohtain the second derivative of the adjoint. flux we differentiate the adjoint transport Eq. (22) twice with respect to $\varepsilon$. Then, noting that the operdior $\left.1^{*} \mid \bar{x}, \Sigma(\bar{x})+\varepsilon \cdot \delta \Sigma(\bar{x})\right\}$ is linear in $\Sigma(\bar{x})$, yielding all its second-or higher-order ierivatives $t o$ be identically zero, that is,

$$
\frac{d^{[1} L\left[\underline{\bar{x}}, \sum(\bar{x})+\varepsilon \cdot \delta \Sigma(\bar{x})\right]}{d \varepsilon}=0, \quad n \geq 2,
$$

we otscain

$\left[\because[\bar{x}, \Sigma(\bar{x})+\varepsilon \cdot \delta \Sigma(\bar{x})] \frac{d^{2} \phi^{*}\left(\bar{x}, \bar{x}_{0}, \Sigma+\varepsilon \cdot \delta \Sigma\right)}{d \varepsilon^{2}}=-2 \quad \frac{d L}{i \dot{x}, \Sigma(\bar{x})+\varepsilon \cdot \delta \Sigma(\bar{x})]} \frac{\mathrm{d} \phi \bar{\phi}^{*}\left(\bar{x}_{,} \bar{x}_{0}, \Sigma+\varepsilon \cdot \delta \Sigma\right)}{\mathrm{d} \varepsilon}\right.$

Taking Eq. (38) at $\varepsilon=0$ and repeating the procedure leading to Eq. (28), ret obtain

$$
\begin{aligned}
& \left.\frac{d^{2} \phi\left(\bar{x}_{0}, \Sigma+\varepsilon \cdot \delta \Sigma\right)}{d \varepsilon^{2}}\right|_{\varepsilon=0}=-\left.\left.2 \int \phi(\bar{x}, \Sigma) \frac{\mathrm{dL}^{*}[\bar{x}, \Sigma(\bar{x})+\varepsilon \cdot \delta \Sigma(\bar{x})]}{d \varepsilon}\right|_{\varepsilon=0} \frac{d \phi^{*}\left(\bar{x}, \bar{x}_{0}, \Sigma+\varepsilon \cdot \delta \Sigma\right)}{d \varepsilon}\right|_{\varepsilon=0} \mathrm{~d} \bar{x} \\
& =-\left.\left.2 \int \frac{\mathrm{d} \phi *\left(\bar{x}, \bar{x}_{0}, \Sigma+\varepsilon \cdot \delta \Sigma\right)}{\mathrm{d} \varepsilon}\right|_{\varepsilon=0} \frac{\mathrm{dL}[\overline{\mathrm{x}}, \Sigma(\overline{\mathrm{x}})+\varepsilon \cdot \delta \Sigma(\overline{\mathrm{x}})]}{\mathrm{d} \varepsilon}\right|_{\varepsilon=0} \phi(\overline{\mathrm{x}}, \Sigma) \mathrm{d} \overline{\mathrm{x}}
\end{aligned}
$$

Our problem now is to find an expression for

$\left.\frac{d \phi\left(\bar{x}, \bar{x}_{0}, \Sigma+\varepsilon \cdot \delta \Sigma\right)}{d \varepsilon}\right|_{\varepsilon=0}$ 
To that goal we recall the meaning of $\phi\left(\bar{x}, \bar{x}_{0}, \Sigma\right)$. It gives the flux at the point $\bar{x}_{0}$ resulting from a unit point source at $\bar{x}$. It will thus be identical to the Green's function $G\left(\bar{x}, \bar{x}_{s}, \Sigma\right)$, which gives the flux at $\bar{x}$ resulting from a unit point source at $\bar{x}_{s}$, and thus is a solution of the equation

$L[\bar{x}, \Sigma(\bar{x})] G\left(\bar{x}, \bar{x}_{s}, \Sigma\right)=\delta\left(\bar{x}-\bar{x}_{s}\right)$

To show this identity, consider Eqs. (22) and (40), from which we see that

$\int G\left(\bar{x}, \bar{x}_{s}, \Sigma\right) \delta\left(\bar{x}-\bar{x}_{0}\right) d \bar{x}=\phi *\left(\bar{x}-\bar{x}_{0}, \Sigma\right) \delta\left(\bar{x}-\bar{x}_{s}\right) d \bar{x}$

yielding

$G\left(\bar{x}_{0}, \bar{x}_{s}, \Sigma\right)=\phi^{*}\left(\bar{x}_{s}, \bar{x}_{0}, \Sigma\right)$

Now looking at Eq. (35) we see that the derivative of the flux at $\bar{x}_{0}$ is expressed in terms of an integral over the unperturbed flux. This suggests that in Eq. (35), $\phi\left(\bar{x}_{0}, \Sigma+\varepsilon \cdot \delta \Sigma\right)$ can be replaced by $G\left(\bar{x}_{0}, \bar{x}_{s}, \Sigma+\varepsilon \cdot \delta \Sigma\right)$ (provided that, in the integral, $\phi(\bar{x}, \Sigma)$ is also replaced by $\left.G\left(\bar{x}_{,} \bar{x}_{s}, \Sigma\right)\right]$ because Eq. (35) is general arnl will hold as long as the fluxes on the left-hand side and the right-hand sidt are solutions of the forward-transport equation with the same source. By doing this and using Eq. (41), we immediately obtain

$$
\left.\frac{\mathrm{d} \phi^{*}\left(\overline{\mathrm{x}}_{2}, \overline{\mathrm{x}}_{0}, \Sigma+\varepsilon \cdot \delta \Sigma\right)}{\mathrm{d} \varepsilon}\right|_{\varepsilon=0}=-\left.\int \phi^{*}\left(\overline{\mathrm{x}}_{1}, \overline{\mathrm{x}}_{0}, \Sigma\right) \frac{\mathrm{dL}\left[\overline{\mathrm{x}}_{1}, \Sigma\left(\overline{\mathrm{x}}_{1}\right)+\varepsilon \cdot \delta \Sigma\left(\overline{\mathrm{x}}_{1}\right)\right]}{\mathrm{d} \varepsilon}\right|_{\varepsilon=0} \mathrm{G}\left(\overline{\mathrm{x}}_{1}, \overline{\mathrm{x}}_{2}, \Sigma\right) \mathrm{d} \overline{\mathrm{x}}_{1},
$$

where for convenience we now index the phase-space variables. (Note that in $\phi^{*}$ the first variable is the unit source location and the second is the field point at which the flux is computed, and in $G$ this is reversed.) Substiluting Eq. (42) into Eq. (39), we obtain 


$$
\begin{aligned}
\left.\frac{d^{2} \phi\left(\bar{x}_{0}, \Sigma+\varepsilon \cdot \delta \Sigma\right)}{d \varepsilon^{2}}\right|_{\varepsilon=0}= & \left.+2 \iint \phi \bar{x}_{1}, \bar{x}_{0}, \Sigma\right)\left.\left.\frac{d L\left(\bar{x}_{1}, \varepsilon\right)}{d \varepsilon}\right|_{\varepsilon=0} G\left(\bar{x}_{1}, \bar{x}_{2}, \Sigma\right) \frac{d L\left(\bar{x}_{2}, \varepsilon\right)}{d \varepsilon}\right|_{\varepsilon=0} \\
& \cdot \phi\left(\bar{x}_{2}, \Sigma\right) \mathrm{d}_{1} \mathrm{~d} \bar{x}_{2}
\end{aligned}
$$

where we again use the shorthand notation $L(x, \varepsilon)$ for $L[\bar{x}, \Sigma(\bar{x})+\varepsilon \cdot \delta \Sigma(\bar{x})]$. Equation (43) gives us an expression for the secont-order perturbation of the flux at $\bar{x}_{0}$ in terms of the unperturbed $f l u x$, the unperturbed Green's function, and differentials of the operator only. Extension to the nth-order term is now quite simple. First, we note from Eq. (24) that

$$
\left.\frac{d^{n} \phi\left(\bar{x}_{0}, \Sigma+\varepsilon \cdot \delta \Sigma\right)}{d \varepsilon^{n}}\right|_{\varepsilon=0}=\left.\int \frac{d^{n} \phi^{*}\left(\bar{x}^{n}, \bar{x}_{0}, \Sigma+\varepsilon \cdot \delta \Sigma\right)}{d \varepsilon^{n}}\right|_{\varepsilon=0} S(\bar{x}) \bar{d} x
$$

Then, from Eq. (38) and using Eq. (37), we can see by induction that the nth derivative of Eq. (38) will yield the recursion equation

$$
L^{*}(x, \varepsilon) \frac{d^{n} \phi^{*}\left(\bar{x}, \bar{x}_{0}, \Sigma+\varepsilon \cdot \delta \Sigma\right)}{d \varepsilon^{n}}=-n \frac{d L *(\bar{x}, \varepsilon)}{d \varepsilon} \frac{d^{n-1} \phi^{*}\left(\bar{x}, \bar{x}_{0}, \Sigma+\varepsilon \cdot \delta \Sigma\right)}{d \varepsilon^{n-1}}
$$

Repeating the procedure of Eq. (28) then yields

$$
\begin{aligned}
\left.\frac{d^{n} g}{d \varepsilon^{n}}\right|_{\varepsilon=0} & =\left.\frac{d^{n} \phi\left(\bar{x}_{0}, \Sigma+\varepsilon \cdot \delta \Sigma\right)}{d \varepsilon^{n}}\right|_{\varepsilon=0} \\
& =-\left.\left.n \int \frac{d^{n-1} \phi^{*}\left(\bar{x}_{n}, \bar{x}_{0}, \Sigma+\varepsilon \cdot \delta \Sigma\right)}{d \varepsilon^{n}}\right|_{\varepsilon=0} ^{\frac{d L\left(\bar{x}_{n}, \varepsilon\right)}{d \varepsilon}}\right|_{\varepsilon=0} \cdot \phi\left(\bar{x}_{n}, \Sigma\right) d \bar{x}_{n}
\end{aligned}
$$

We now proceed by finite induction assuming that

$$
\left.\frac{\mathrm{d}^{\mathrm{n}-1} \phi\left(\bar{x}_{0}, \Sigma+\varepsilon \cdot \delta \Sigma\right)}{\mathrm{d} \varepsilon^{n-1}}\right|_{\varepsilon=0}=\left.(-1)^{\mathrm{n}-1}(\mathrm{n}-1) ! \int \ldots \int \phi^{*}\left(\overline{\mathrm{x}}_{1}, \overline{\mathrm{x}}_{0}, \Sigma\right) \frac{\mathrm{dL}\left(\overline{\mathrm{x}}_{1}, \varepsilon\right)}{\mathrm{d} \varepsilon}\right|_{\varepsilon=0} \mathrm{G}\left(\overline{\mathrm{x}}_{1}, \overline{\mathrm{x}}_{2}\right)
$$




$$
\left.\left.\frac{d L\left(\bar{x}_{2}, \Sigma\right)}{d \varepsilon}\right|_{\varepsilon=0} G\left(\bar{x}_{2}, \bar{x}_{3}\right) \ldots G\left(\bar{x}_{n-2}, \bar{x}_{n-1}\right) \frac{d L\left(\bar{x}_{n-1}, \varepsilon\right)}{d \varepsilon}\right|_{\varepsilon=0} \phi\left(\bar{x}_{n-1}, \Sigma\right) d \bar{x}_{1} \ldots d \bar{x}_{n-1}
$$

and then, repeating the procedure of Eqs. (40)-(42), replacing $\phi\left(\bar{x}_{0}, \Sigma\right)$ by $G\left(\bar{x}_{0}, \bar{x}_{n}\right)$ and $\phi\left(\bar{x}_{n-1}, \Sigma\right)$ by $G\left(\bar{x}_{n-1}, \bar{x}_{n}\right)$, and using the identity of the Green': function and the adjoint, we get

$$
\left.\frac{d^{n-1} \phi^{*}}{d \varepsilon^{n-1}} \frac{\left(\bar{x}_{n}, \bar{x}_{0}, \Sigma+\varepsilon \cdot \delta \Sigma\right)}{\varepsilon=0}\right|_{0}=\left.(-1)^{n-1}(n-1) ! \int \ldots \int \phi^{*}\left(\bar{x}_{1}, \bar{x}_{0}, \Sigma\right) \frac{d L\left(\bar{x}_{1}\right)}{d \varepsilon}\right|_{\varepsilon=0} G\left(\bar{x}_{1}, \bar{x}_{2} ;\right.
$$

$$
\left.\ldots \frac{d L\left(\bar{x}_{n-1}, \varepsilon\right)}{d \varepsilon}\right|_{\varepsilon=0} G\left(\bar{x}_{n-1}, \bar{x}_{n}\right) d \bar{x}_{1} \ldots d \bar{x}_{n-1}
$$

Substituting Eq. (48) into (46) we finally obtain the nth-order derivative it the flux in the form

$$
\begin{array}{r}
\left.\frac{d^{n} g}{d \varepsilon^{n}}\right|_{\varepsilon=0}=\left.\frac{d^{n} \phi\left(\bar{x}_{0}, \Sigma+\varepsilon \cdot \delta \Sigma\right)}{d \varepsilon^{n}}\right|_{\varepsilon=0}=\left.(-1)^{n} \int_{n !} \bar{x}_{1} \ldots \int_{x_{n}^{*} \phi^{*}\left(\bar{x}_{1}, \bar{x}_{0}, \Sigma\right)} \frac{d L\left(\bar{x}_{1}, \varepsilon\right)}{d \varepsilon}\right|_{\varepsilon=0} G\left(\bar{x}_{1}, \bar{x}_{2}\right) \\
\\
\left.\quad \ldots G\left(\bar{x}_{n-1}, \bar{x}_{n}\right) \frac{d L\left(\bar{x}_{n}, \varepsilon\right)}{d \varepsilon}\right|_{\varepsilon=0} \phi\left(\bar{x}_{n}, \Sigma\right) d \bar{x}_{1} \ldots d \bar{x}_{n} .
\end{array}
$$

Because the assumption [Eq. (47)] was true for $n=1,2$, Eq. (49) holds for every n. Now substituting Eq. (49) in the Taylor series of Eq. (34), we get

$\delta \phi\left(\bar{x}_{0}\right)=\phi\left(\bar{x}_{0}, \Sigma+\delta \Sigma\right)-\phi\left(\bar{x}_{0}, \Sigma\right)=\sum_{n=0}^{\infty}(-1)^{n} \Gamma_{n}$, 
where $\Gamma_{n}$ is extracted from Eqs. (34) and (49). This can be written in a more concise form as

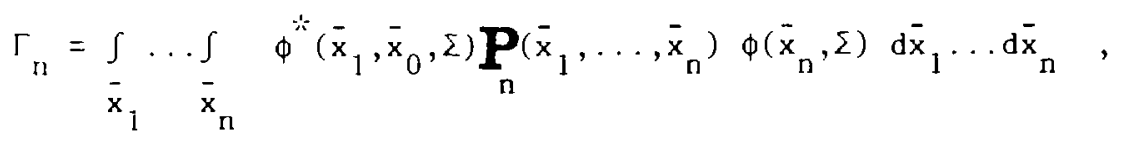

with $\mathbf{P}_{n}\left(\bar{x}_{1}, \ldots, \bar{x}_{n}\right)$ bejng an n-dimensional operator referred to as the nth orier perturbation operator

$$
\mathbf{P}_{n}\left(\bar{x}_{1}, \ldots, x_{n}\right)=\left.\left.\frac{\left.d I \cdot \mid \bar{x}_{1}, \Sigma\left(\bar{x}_{1}\right)+\varepsilon \cdot \delta \Sigma\left(\bar{x}_{1}\right)\right]}{d \varepsilon}\right|_{\varepsilon=0} \prod_{i=2}^{n} G\left(\bar{x}_{i-1}, \bar{x}_{1}\right) \frac{d L\left[\bar{x}_{i}, \Sigma\left(\bar{x}_{i}\right)+\varepsilon \cdot \delta \Sigma\left(\bar{x}_{i}\right)\right]}{d \varepsilon}\right|_{\varepsilon=0}
$$

This concludes the proof that the perturbation can be developed into ars infinite explicit series such that each term depends on the unperturbed flux and Green's function, and on the differential of the operator, which will eventually be the perturbation in the data field. The folding of this series with the detector response will yield, as was already shown, a first ierm identical to first-order perturbation theory. To show that the higher-order terms are a natural extension of the first-order term, we now give an intuitive derivation of the series, which may also give some insight into its physical meaning.

Consider the steady-state situation before the perturbation is introduced. Here, the flux at each point $\bar{x}_{0}$ is a solution of

$L\left(\bar{x}_{0}, \Sigma\right) \phi\left(\bar{x}_{0}, \Sigma\right)=S\left(\bar{x}_{0}\right)$

A perturbation $\Delta \mathrm{L}\left(\bar{x}_{0}, \delta \Sigma\right)$ is introduced such that $\mathrm{L} \rightarrow \mathrm{L}+\Delta \mathrm{L}$. This situation can be visualized as if, at each point $\bar{x}$, an additional source of magnitude - $\Delta \mathrm{L} \phi(\overline{\mathrm{x}}, \Sigma)$ were introduced. This in return will cause a first-order change of the flux at each point $\bar{x}_{0}$, given by

$\delta_{1} \phi\left(\overline{\mathrm{x}}_{0}\right)=-\int \phi^{\ddot{*}}\left(\overline{\mathrm{x}}_{1}, \overline{\mathrm{x}}_{0}\right) \Delta \mathrm{L} \phi\left(\overline{\mathrm{x}}_{1}, \Sigma\right) \mathrm{d} \overline{\mathrm{x}}_{1}$. 
In the next stage, $\phi\left(\bar{x}_{1}, \Sigma\right)$ in Eq. (54) has an increment $\delta_{1} \phi\left(\bar{x}_{1}, \Sigma\right)$. when substituted in Eq. $(54), \phi\left(\bar{x}_{1}, \Sigma\right) \longrightarrow \phi\left(\bar{x}_{1}, \Sigma\right)+\delta_{1} \phi\left(\bar{x}_{1}\right)$ and the equation yields the second-order change

$\delta_{2} \phi\left(\bar{x}_{0}\right)=+\int \phi^{*}\left(\bar{x}_{1}, \bar{x}_{0}\right) \Delta \mathrm{L}\left(\mathrm{x}_{1}\right) \phi^{*}\left(\bar{x}_{2}, \bar{x}_{1}\right) \Delta \mathrm{L}\left(\bar{x}_{2}\right) \phi\left(\bar{x}_{2}, \Sigma\right) d \bar{x}_{1} \mathrm{~d} \bar{x}_{2}$

Repeating this process in which a given-order prerturbation iteratively causes a next higher-order perturbation will yield the same expansion as in Eq. (50).

Returning now to the change of the detector response funclional $R$, consin. the important case where $R$ is linear in $\phi(\bar{x})$ and $\Sigma_{r}(\bar{x})$; that is,

$F\left[\phi(\bar{x}), \Sigma_{r}(\bar{x})\right]=\phi(\bar{x}) \Sigma_{r}(\bar{x})$

In this case, referring to the Taylor series expansion of $R$ [Eq. (9)], one , in easily find that the nth-order term is given by

$$
\begin{aligned}
\left.\frac{1}{n !} \frac{d^{n} f(\varepsilon)}{d \varepsilon^{n}}\right|_{\varepsilon=0} & =\left.\frac{1}{n !} \frac{d^{n} R(\Sigma+\varepsilon \cdot \delta \Sigma)}{d \varepsilon^{n}}\right|_{\varepsilon=0} \\
& =\left.\frac{1}{n !} \int_{D} n \delta \Sigma_{r}\left(\bar{x}_{0}\right) \frac{d^{n-1} \phi\left(\bar{x}_{n}, \Sigma+\varepsilon \cdot \delta \Sigma\right)}{d \varepsilon^{n-1}}\right|_{\varepsilon=0}+\Sigma_{r}\left(\bar{x}_{0}\right)-\left.\frac{d^{n} \phi\left(\bar{x}_{0}, \Sigma+\varepsilon \cdot \delta \Sigma\right)}{d \varepsilon^{n}}\right|_{\varepsilon=0} \cdot \bar{x}_{0}
\end{aligned}
$$

Substitution of the terms developed for the flux into Eq. (57) is straight forward. The first term will yield the first-order perturbation (includirg the direct and indirect effects). In each successive term the integration over $\bar{x}_{0}$ will transfer the adjoint flux $\phi \bar{\phi}^{*}\left(\bar{x}_{1}, \bar{x}_{0}\right)$ into the usual adjoint $\phi^{*}\left(\bar{x}_{1}\right)$. Equation (57) combined with the Taylor series for the flux gives a general expression for the change in any target response. Knowledge of the detailed Green's function of the system and the unperturbed flux enables the calculation of the change in the target response to any desired degree of accuracy.

† Note that $\Sigma_{r}(\bar{x})$ is the detector response function and $\delta \Sigma(\bar{x})$ may or may not be equal to $\delta \Sigma_{r}(\bar{x})$. 


\section{v. CONCLUSIONS}

A differential approach to sensitivity analysis has been developed. The approach leads to the expansion of the perturbation in a Taylor series wher the first-order term coincides with first-order perturbation theory results. A general explicit expression has been derived for the nth-order term that utilizes the unperturbed flux, adjoint flux, and a detailed Green's functjon, all from the unperturbed problem, everywhere in the region of the perturhation." One may note that once the Green's function $G(\bar{x}, \bar{y})$ is defined for every paur $(\bar{x}, \bar{y})$ within the perturbed region, the perturbation can be calculated to dny degree of accuracy. It is interesting that, therefore, the additional information required to calculate the second-order term will suffice $t: 1$ calculate any higher-order term. However, the calculation of $G(\bar{x}, \bar{y})$ is in no way trivial and may require no less effort than a direct calculation if the perturbed target response. In some situations it may be possible to approximate $G(\bar{x}, \bar{y})$ analytically, as in a highly absorbing region, and use this approximation to evaluate the serond-order and higher terms. Because the expansion is infinite, we are not limited to small perturbations.

As can be seen by an examination of Eq. (55), the second-order term 1 s naturally nonlinear in $\delta \mathrm{L}$, unlike a first-order perturbation as in Eq. (3i). In the usual application to sensitivity analysis $\delta L(\bar{x})$ is held constant over a spatial region and energy group, that is, a region of phase space, $S$. For example. in cross-section sensitivity,

$\delta L(\bar{x})=\delta \Sigma(\bar{x})=\left\{\begin{array}{lll}\delta \Sigma & , \bar{x} \varepsilon s \\ 0, & \bar{x}<s\end{array}\right.$,

and $\delta \Sigma_{r}$ is a constant in $S$. The second-order sensitivity is then, from $E q$. (55), linear in $\delta \Sigma$.

$\frac{\delta_{2} \phi\left(\bar{x}_{0}\right)}{\delta \Sigma_{s}}=\delta \Sigma s \iint_{S} \phi^{*}\left(\bar{x}_{1}, \bar{x}_{0}\right) \phi^{*}\left(\bar{x}_{2}, \bar{x}_{1}\right) \phi\left(\bar{x}_{2}, \Sigma\right) d \bar{x}_{1} \mathrm{dx} \bar{x}_{2}$.

'The role of a detailed Green's function in calculating higher-order sensitivity terms was realized by T. E. Albert. ${ }^{10}$ 
Similarly, it can be seen from Eq. (52) that higher-order terms will involves successively higher powers of $\delta \Sigma_{\mathrm{S}}$, $i n$ the form

$$
\frac{\delta_{n} \phi\left(\bar{x}_{0}\right)}{\delta \Sigma \Sigma_{s}}=(\delta \Sigma)^{n-1} \int_{\bar{x}_{1} \varepsilon S} \ldots \int_{\bar{x}_{n} \varepsilon S} \phi *\left(\bar{x}_{1}, \bar{x}_{0}\right) \phi\left(\bar{x}_{n}\right) \prod_{i=2}^{n} \phi \prod^{*}\left(\bar{x}_{i}, \bar{x}_{i-1}\right) d \bar{x}_{1} \ldots d \bar{x}_{n},
$$

where $\phi^{t}\left(\bar{x}_{i}, \bar{x}_{i-1}\right)$ is the unperturbed adjoint Green's function. It cani ther be seen that for $n>1$, a superposition of operator perturbations is nn, longer possible, as it was in first-order sensitivity. That is, if

$$
\delta \mathrm{L}=\sum_{\mathrm{k}} \delta \mathrm{L}_{\mathrm{k}},
$$

then $\delta_{n} \phi\left(\bar{x}_{0}\right) / \delta L$ will involve cross terms $\delta L_{i} \delta L_{j}$ Specifically, wher: $\delta L=\delta \Sigma_{s}=\sum_{k} \delta \Sigma_{s, k}$, a set of partial cross-sections, the total cross-secturn sensitivity is obviously no longer a simple sum of the partial sensitivitics.

\section{ACKNOWLEDGEMENT}

The authors are pleased to acknowledge many stimulating discussiom regarding this topic with Edward W. Larsen of Los Alamos National Laboratory

\section{REFERENCES}

1. E. M. Oblow, "Sensitivity Theory from a Differential Viewpoint," Nucl. Sri. Eng. 59, 187-189 (1976); and Corrigendum, Nucl. Sci. Eng. 65, 428 (1978).

2. D. R. Smith, Variational Methods in Optimization, (Prentice-Hall, Inc.. Englewood Cliffs, N. J., 1974), p.18.

3. G. C. Evans, Functionals and Their Applications, (Dover Publications, Inc., New York, 1964), p. 1 .

4. V. Volterra, Theory of Functionals, (Dover-Publications, Inc., New York, 1959), p.22.

5. D. R. Smith, Variational Methods in Optimization, (Prentice-Hall, Inc., Englewood Cliffs, N. J., 1974) pp.23, 35. 
6. D. R. Smith, Variational Methods in Optimization, (Prentice-Hall, Inc., Englewood Cliffs, N. J., 1974), P.55.

7. V. Volterra, Theory of Functionals, (Dover Publications, Inc., New York, 1959), p.26.

8. D. R. Smith, Variational Methods : Optimization, (Prentice-Hall, Inc.. Englewood Cliffs, N. J., 1974), p. 290 .

9. S. A. W. Gerstl and W. M. Stacey, Jr., "A Class of Second-Order Approximat, Formulations of Deep Penetration Radiation Transport Problems, "Nucl. Sil Eng. 51, 339 (1973).

10. T. E. Albert, "Introduction of the Green's Function in Cross-sectum Sensitivity Analysis," Trans. Am. Nucl. Soc. 17, $546\left(2, i_{2}\right.$; 